\title{
Mib-1 proliferation index is an independent predictor of lymph node metastasis in invasive breast cancer: A prospective study on 675 patients
}

\author{
DANIELE MARRELLI ${ }^{1}$, ENRICO PINTO $^{1}$, ALESSANDRO NERI $^{1}$, TIZIANA MEGHA ${ }^{2}$, WALTER GIOFFRË ${ }^{4}$, \\ TOMMASO CIOPPA $^{1}$, GIOVANNI DE MARCO ${ }^{1}$, ALFONSO DE STEFANO ${ }^{1}$ and FRANCO ROVIELLO ${ }^{3}$
}

Departments of ${ }^{1}$ General Surgery and Surgical Oncology, ${ }^{2}$ Human Pathology and Oncology and ${ }^{3}$ General Surgery and Surgical Oncology, Unit of Advanced Surgical Oncology, University of Siena; ${ }^{4}$ Unit of Senology, University of Siena, Italy

Received July 18, 2005; Accepted September 16, 2005

\begin{abstract}
In order to evaluate the potential risk factors for Lymph node metastasis in invasive breast cancer patients submitted to axillary dissection, 675 patients who received surgery between January 1995 and December 2002 were included in a prospective study. In all cases, MIB-1 proliferation index was investigated by immunohistochemistry. Lymph node involvement was found in 248 out of 675 patients. Univariate analysis showed that peritumoral lymphovascular invasion, pT stage, tumor multiplicity, MIB-1 proliferation index $>10 \%$, oestrogen receptor status, histological type, tumor grade and progesterone receptor status were related to a higher incidence of Lymph node metastasis, with various levels of statistical significance. Multivariate analysis identified lymphovascular invasion [relative risk (RR, 7.69; $\mathrm{p}<0.001)$, pT stage (RR, 3.08; p<0.001), tumor multiplicity (RR, 3.89; $\mathrm{p}<0.001$ ), and MIB-1 proliferation index ( $R R, 1.66$; $\mathrm{p}=0.019)$ ] as independent predictive variables. The impact of MIB-1 positivity on the incidence of Lymph node metastasis was particularly evident in intermediate risk groups (pT1c, pT2 without lymphovascular invasion), as well as in grade-2 tumors. In conclusion, the MIB-1 proliferation index could provide additional information about the risk of Lymph node metastasis in invasive breast cancer, and may be useful to identify grade- 2 tumors with a more aggressive clinical behaviour.
\end{abstract}

\section{Introduction}

Nodal status is a strong prognostic factor in breast cancer and is one of the most important variables considered in deciding on adjuvant treatment after surgery. Sentinel node biopsy,

Correspondence to: Dr Daniele Marrelli, Department of General Surgery and Surgical Oncology, University of Siena, Viale Bracci, 53100 Siena, Italy

E-mail: Marrelli@unisi.it

Key words: breast cancer, lymph node metastasis, lymphadenectomy, MIB-1, multivariate analysis, proliferation index although not the standard of care, is rapidly becoming widely accepted as an alternative staging procedure for the axilla in breast cancer. However, in subgroups of patients with a high risk of Lymph node metastasis, this procedure may not provide a clinical benefit and may involve a waste of resources as, in a high percentage of cases, it is followed by axillary dissection. The definition of tumor characteristics that are predictive of Lymph node metastasis may be useful in order to better select which patients to submit to axillary surgery (1).

Besides commonly used pathological factors, cell proliferation has an important role as an indicator of biological aggressiveness in breast cancer. Ki-67 antibody reacts with a nuclear antigen which is present in G1, S, G2 phases and mitosis but is absent in G0 and is therefore considered a marker of the proliferative activity of the tumor (2). Unfortunately, the epitope that recognizes $\mathrm{Ki}-67$ is destroyed in the fixation and paraffin embedding process. More recently, the MIB-1 antibody, which recognizes Ki-67 antigen in formalin-fixed, paraffin-embedded tissue, has been described and applied to breast cancer $(3,4)$.

Several studies have investigated the role of the proliferation index in breast cancer patients and a strong correlation between the MIB-1 labelling index and Lymph node metastasis has been reported $(5,6)$, although this finding has not been confirmed by others (7-10). Similarly, conflicting results regarding the prognostic significance of the proliferation index have been found $(9,11-13)$.

This prospective study was designed with the aim of evaluating the potential risk factors for Lymph node metastasis in a consecutive series of invasive breast cancer patients submitted to axillary dissection, with special reference to the role of the MIB-1 labelling index. Surgical treatment, histopathological examination and immunohistochemistry were performed on the entire series according to standard criteria.

\section{Patients and methods}

Patients. For this prospective observational study, we considered 675 patients with invasive breast cancer who received surgery at the Department of General Surgery and Surgical Oncology, University of Siena, between January 1995 and December 2002. The mean ( \pm standard deviation, SD) 
age of the patients was $62 \pm 13$ years (range, 27-94). Patients who underwent neoadjuvant chemotherapy and patients with inflammatory carcinoma were excluded from the study. Surgical treatment consisted of radical mastectomy in 309 patients and quadrantectomy in 366 patients. Only patients submitted to complete axillary dissection of first and second level lymph nodes were included; in the entire series, a mean (SD) of $18 \pm 6$ lymph nodes (range, 7-50) were removed.

Pathological examination. After surgical removal, tumor specimens were fixed in formalin and sent for histopathological examination. Standard histopathological examination of axillary nodes included one central section of each lymph node, stained with haematoxylin and eosin. The histological type was defined as 'ductal', 'lobular' or 'other', according to the common histological criteria of the WHO classification. Histological grading was classified as well differentiated (grade 1), moderately differentiated (grade 2), or poorly differentiated (grade 3,) according to the Bloom and Richardson criteria modified by Elston and Ellis (14). The pathological tumor stage was assessed according to the criteria established by the International Union Against Cancer (UICC) (6th edition) (15). Oestrogen (ER) and progesterone receptor status (PGR) were assessed by immunohistochemistry and classified as 'negative' or 'positive'. Both multifocal and multicentric tumors were included in the 'multiplicity' subgroup (16).

MIB-1 immunostaining. Paraffin sections were cut to a thickness of five micrometers, immersed in citric acid buffer (pH 6.0) and incubated in a microwave oven twice at $750 \mathrm{~W}$ for $5 \mathrm{~min}$. The sections were subsequently immunostained using the APAAP Complex method (Dako, Denmark) with the monoclonal antibody, MIB-1 (Dako). The intensity of immunostaining per cell was not quantified. Tonsil samples from our routine files were used as positive controls. For negative controls, we used normal mouse serum in which the primary antibody had been prepared.

Cells that stained positive for MIB-1 were registered in 10 randomly chosen HPFs and expressed as percentages of all epithelial cells. The slides were scored by the same pathologist (T.M.). We considered the tumor positive for MIB-1 when $\geq 10 \%$ of the cells counted were stained.

Statistical analysis. Correlation between Lymph node status (negative, positive) and clinicopathological variables was investigated by means of univariate and multivariate analysis. The $\chi^{2}$ test was used to assess the statistical significance of the association between categorical variables and incidence of Lymph node metastasis or MIB-1 positivity. Differences in continuous variables were evaluated using the analysis of variance (ANOVA) test.

A logistic regression model was built in order to identify the variables that influenced Lymph node status in an independent manner, by multivariate analysis (17). The presence of Lymph node metastasis was considered as a dependent variable, whereas the following were considered as covariates: age (continuous variable), tumor grade $(1,2,3)$, histological type (ductal, lobular, others), tumor multiplicity (absent, present), ER status (negative, positive), PGR status (negative, positive), pT stage (pT1a-pT1b, pT1c, pT2, pT3-pT4), number of
Table I. Incidence of axillary lymph node metastasis according to variables under study.

\begin{tabular}{lrrrr}
\hline Variable & \multicolumn{3}{c}{ No. of Lymph node Lymph node p-value } \\
& patients & negative & \multicolumn{1}{c}{ positive } & \\
\hline Age (mean \pm SD) & & $63 \pm 12$ & $62 \pm 13$ & 0.353 \\
Tumor grade & & & & \\
1 & 148 & $103(70)$ & $45(30)$ & 0.039 \\
2 & 331 & $210(63)$ & $121(37)$ & \\
3 & 170 & $95(56)$ & $75(44)$ & \\
Unknown & 26 & & & \\
Histological type & & & & \\
$\quad$ Ductal & 512 & $320(63)$ & $192(37)$ & 0.007 \\
Lobular & 96 & $54(56)$ & $42(44)$ & \\
Others & 67 & $53(79)$ & $14(21)$ &
\end{tabular}

Tumor

multiplicity

Absent
Present

567
108

$390(69)$
$37(34)$

$177(31)<0.001$

ER status

Negative

Positive

Unknown

$\begin{array}{rr}161 & 84(52) \\ 511 & 342(67) \\ 3 & \end{array}$

$77(48)$

$169(33)$

0.001

PGR status

$\begin{array}{lrrrr}\text { Negative } & 203 & 117(58) & 86(42) & 0.043 \\ \text { Positive } & 469 & 309(66) & 160(34) & \\ \text { Unknown } & 3 & & \end{array}$

No. of removed

lymph nodes

$($ mean $\pm \mathrm{SD})$

Lymphovascular

invasion

\begin{tabular}{lrcrl} 
Absent & 503 & $376(75)$ & $127(25)$ & $<0.001$ \\
Present & 146 & $34(23)$ & $112(77)$ & \\
Unknown & 26 & & & \\
pT stage & & & & \\
pT1a-pT1b & 160 & $140(88)$ & $20(12)$ & $<0.001$ \\
pT1c & 321 & $225(70)$ & $96(30)$ & \\
pT2 & 155 & $59(38)$ & $96(62)$ & \\
pT3-pT4 & 39 & $3(8)$ & $36(92)$ & \\
MIB-1 expression & & & & \\
Negative $(\leq 10 \%)$ & 357 & $251(70)$ & $106(30)$ & $<0.001$ \\
Positive $(>10 \%)$ & 318 & $176(55)$ & $142(45)$ & \\
\hline
\end{tabular}

Numbers in parentheses are percentages; SD, standard deviation; ER, oestrogen receptor; PGR, progesterone receptor.

removed lymph nodes (continuous variable), peritumoral lymphovascular invasion (LVI) (absent, present), and MIB-1 proliferation index $(\leq 10 \%,>10 \%)$.

In the statistical program, the parameters of the model were estimated using the maximum-likelihood method. Significant variables were included in the model by means of forward stepwise selection: starting with a model containing 
Table II. Independent predictors of lymph node metastasis (logistic regression model).

\begin{tabular}{lcc}
\hline Variable & p-value & Hazard ratio $(95 \% \mathrm{CI})$ \\
\hline Lymphovascular invasion & $<0.001$ & $7.69(4.60-12.85)$ \\
pT stage & $<0.001$ & $3.08(2.29-4.15)$ \\
Tumor multiplicity & $<0.001$ & $3.89(2.19-6.88)$ \\
MIB-1 positivity & 0.019 & $1.66(1.08-2.54)$ \\
\hline
\end{tabular}

CI, confidence interval.

only the constant, at each step the variable with the smallest significance value entered the model, with a default level of $\mathrm{p}<0.05$. The significance value of each factor was reassessed at each step; if a variable in a forward stepwise block exceeded a significance level of 0.1 , it was removed from the model. Removal testing was based on the probability of the likelihood-ratio statistic. The Statistical Package for the Social Sciences software (version 11.0) (SPSS ${ }^{\mathrm{TM}}$, Chicago, IL, USA) was used for statistical analysis.

\section{Results}

Lymph node involvement was found in 248 out of 675 patients $(36.7 \%)$. In the 248 positive cases, the mean (SD) number of positive lymph nodes was $6.8 \pm 6.7$ (range, 1-36).

By immunohistochemistry, MIB-1 positivity ( $>10 \%$ of tumor cells) was detected in 318/675 cases (47.1\%). MIB-1 positivity was significantly related to tumor grade $(\mathrm{p}<0.001)$, pT stage $(p<0.001)$, Lymph node involvement $(p<0.001)$, negative ER status $(\mathrm{p}<0.001)$, negative PGR status $(\mathrm{p}<0.001)$ and ductal histological type $(\mathrm{p}<0.005)$.

The correlation between the incidence of Lymph node metastasis and the variables studied is reported in Table I; statistical analysis revealed a significant association with tumor grade, histological type, tumor multiplicity, ER status, PGR status, LVI, pT stage, and MIB-1 positivity, with various levels of statistical significance. The highest incidence of Lymph node metastasis was found in pT3-pT4 and pT2 tumors (92\% and 62\%, respectively), in cases with LVI (77\%) and when tumor multiplicity was present (66\%). MIB-1-negative cases showed positive lymph nodes in $30 \%$ of patients, in comparison to $45 \%$ of MIB-1-positive cases $(p<0.001)$.

The influence of different variables on the risk of Lymph node metastasis was studied by means of multivariate analysis (logistic regression model), and the results are reported in Table II. LVI, pT stage, tumor multiplicity and MIB-1 positivity were selected as independent predictive variables. All other variables under study were excluded from the model. Total $\chi^{2}$ of the model at step number 4 was 236.414 , with a statistical significance of $\mathrm{p}<0.001$, and residual $\chi^{2}$ was 8.785 ( $p=0.553)$, thus indicating that the model fits the data more than adequately.

In order to evaluate the potential clinical utility of the MIB-1 labelling index in identifying patients at risk of Lymph node metastasis, a further subgroup analysis was performed using pT stage, LVI and MIB-1 expression (Table III); this
Table III. Incidence of axillary lymph nodes metastasis according to $\mathrm{pT}$ stage, peritumoral lymphovascular invasion, and MIB-1 expression.

\begin{tabular}{lcccccc}
\hline pT stage & $\begin{array}{c}\text { Lympho- } \\
\text { vascular } \\
\text { invasion }\end{array}$ & $\begin{array}{c}\text { MIB-1 } \\
\text { negative }\end{array}$ & $\begin{array}{c}\text { MIB-1 } \\
\text { positive }\end{array}$ & p-value \\
\hline pT1a-pT1b & & & & & & \\
& Absent & $10 / 103$ & $(10)$ & $3 / 38$ & $(8)$ & 0.737 \\
& Present & $4 / 10$ & $(40)$ & $3 / 7$ & $(43)$ & 0.906 \\
pT1c & & & & & & \\
& Absent & $20 / 122$ & $(16)$ & $30 / 120$ & $(25)$ & 0.097 \\
& Present & $12 / 23$ & $(52)$ & $26 / 33$ & $(79)$ & 0.036 \\
pT2 & & & & & & \\
& Absent & $17 / 43$ & $(40)$ & $37 / 65$ & $(57)$ & 0.076 \\
& Present & $19 / 20$ & $(95)$ & $23 / 27$ & $(85)$ & 0.261 \\
pT3 - pT4 & & & & & & \\
& Absent & $5 / 5$ & $(100)$ & $5 / 7$ & $(71)$ & 0.118 \\
& Present & $13 / 14$ & $(93)$ & $12 / 12$ & $(100)$ & 0.259 \\
\hline
\end{tabular}

Numbers in parentheses represent the incidence of lymph node metastasis.

time, tumor multiplicity was excluded from the analysis because it is difficult to assess by routine preoperative diagnostic examination and excisional biopsy. A strong influence of LVI on the incidence of Lymph node metastasis was found in all subgroups, with the exception of pT3-pT4 tumors; in patients with peritumoral LVI, very high rates of nodal metastasis were found at pT1a-pT1b stage (41\%), pT1c stage $(68 \%)$ and pT2 stage $(89 \%)$. In contrast, pT1a-pT1b tumors without LVI showed a low risk of Lymph node metastasis (9\%), irrespective of MIB-1 expression. With respect to MIB-1 positivity, this factor influenced the risk of nodal metastases in pT1c stage with LVI (52\% in MIB-1negative cases, vs. 79\% in MIB-1-positive cases, $\mathrm{p}<0.05)$. A similar trend was also observed in pT1c stage tumors without LVI, and in pT2 stage tumors without LVI, although this was not statistically significant.

During analysis of the subgroups, we also noticed a strong impact of MIB-1 immunoreactivity on the incidence of nodal metastasis when stratifying for tumor grade (Table IV). A significant difference related to MIB-1 positivity was observed in grade- 2 tumors $(27 \%$ vs. $50 \%, \mathrm{p}<0.001)$; those with negative MIB-1 expression had an incidence of Lymph node metastasis similar to grade-1 tumors, whereas in MIB-1-positive cases the incidence was similar to grade- 3 tumors.

\section{Discussion}

Prediction of lymph node status is an important issue in breast cancer patients, and several papers in the literature are concerned with both commonly used and non-conventional predictive factors $(1,16,18,19)$. Several authors have focused their attention on molecular markers identified by immunohistochemistry $(5-7,9)$. However, many studies are retrospective, and often include patients operated on over a long 
Table IV. Incidence of axillary lymph node metastasis according to tumor grade and MIB-1 expression.

\begin{tabular}{lcccc}
\hline Tumor grade & MIB-1 negative & \multicolumn{2}{c}{ MIB-1 positive } & p-value \\
\hline 1 & $27 / 102(26)$ & $18 / 46 \quad(39)$ & 0.126 \\
2 & $54 / 197(27)$ & $67 / 134(50)$ & $<0.001$ \\
3 & $22 / 47 \quad(47)$ & $53 / 123(43)$ & 0.663 \\
\hline
\end{tabular}

Numbers in parentheses represent the incidence of lymph node metastasis.

time period or in different surgical units. This study was designed in a prospective manner. All patients were operated on in the same Surgical Department, using a standard technique for axillary Lymph node dissection. The resected specimens were processed in the Pathological Unit according to standard procedure, and immunohistochemical analysis was conducted and evaluated by the same pathologists, with the aim of minimizing the potential bias related to different methodological techniques and inter-observer reproducibility, which is commonly found in immunohistochemistry (20). Of the different methods for estimating cell proliferation, we employed MIB-1 expression because of its simplicity in comparison to $\mathrm{Ki}-67$ analysis, which requires frozen material and a more complex procedure (21); furthermore, a better clinical significance of MIB-1 labelling index on paraffin sections has been reported with respect to $\mathrm{Ki}-67$ expression on frozen material (4).

Similarly to the results of other studies, LVI and pT stage were the most important predictors of Lymph node metastasis in our patients. The incidence of Lymph node metastasis increases with the pT stage $(1,18)$. This was confirmed in our series, in which only $12 \%$ of pT1a or pT1b lesions were $\mathrm{N}$-positive. However, even in this low-risk group, the few cases (17 out of 158, 11\%) with LVI had an incidence of Lymph node metastasis higher than $40 \%$. The effect of LVI on nodal status was also evident in pT1c and pT2 tumors, a finding reported by other authors and in a previous study by our group $(6,19,22)$. The strong association with Lymph node metastasis indicates that LVI could probably be regarded as the precursor of nodal involvement (23). Peritumoral vascular invasion has also been reported as a risk factor for additional axillary metastases in patients with a positive sentinel lymph node, which indicates a greater propensity to spread to multiple lymph nodes (24). As such, LVI requires careful consideration when deciding about surgical approach to the axilla.

The results of univariate and multivariate analysis showed that MIB-1 expression could provide additional information about the risk of axillary Lymph node metastasis. The effect of MIB-1 expression is particularly evident in the intermediate risk groups (pT1c, pT2 without LVI), which may increase its clinical utility. The incidence of nodal metastases in pT1c tumors with LVI and MIB-1 positivity was $79 \%$, which is similar to that of the highest risk groups. On the contrary, the risk in MIB-1-negative pT2 tumors without LVI was lower with respect to smaller tumors (pT1a-pT1b) with LVI. No effect was found in very low or very high risk groups (pT1apT1b, pT2 with LVI, and pT3-pT4).

In addition to Lymph node status, MIB-1 positivity was significantly related to tumor grade, $\mathrm{pT}$ stage, negative ER and PGR status, and ductal histological type; no correlation was found with LVI. On the other hand, both MIB-1 positivity and LVI were independent risk factors for Lymph node metastasis. This suggests that a greater proliferative activity of tumor cells may not affect the invasion of peritumoral lymphatic vessels but may increase the propensity to spread to axillary lymph nodes in an independent manner. In a recent study, a greater proliferation index was found in positive axillary lymph nodes with respect to their primary breast tumors, thus suggesting that cells with a more aggressive potential (such as those with a high proliferation index) are more likely to escape from the primary tumor and metastasize to axillary lymph nodes (25).

The association between MIB-1 labelling index and Lymph node metastasis in breast cancer patients has been reported by other authors, mostly when early stages of invasive breast cancer were considered $(5,6)$. In other studies, on the contrary, the proliferation index was found to be related to an adverse prognosis but it did not correlate with Lymph node status, although a multivariate analysis by means of a prediction model was rarely performed $(8-10,13)$. Differences in patient cohorts, immunohistochemical technique, inter-observer variability, counting method and the cut-off level used may explain the different results (20). We believe that a prospective study conducted on a large series in a single institution, with a standard technique and a specifically designated pathologist for immunohistochemical analysis, may be one of the best methods for assessment of the clinical significance of molecular markers in breast cancer.

Even though tumor multiplicity was a strong predictor of Lymph node metastasis with a high relative risk at multivariate analysis, we did not include this factor in the subgroup analysis because its definition by routine preoperative diagnostic examination and excisional biopsy is difficult. However, when we examined the group of patients without tumor multiplicity separately, the impact of MIB-1 expression on the risk of Lymph node metastasis was confirmed (data not shown). The influence of tumor multiplicity on axillary status has been reported in several studies (26). This may be due to a higher tumor volume in multicentric or multifocal tumors, where pT stage is classified according to the size of the dominant lesion, with respect to unifocal lesions. Recent studies have suggested, however, that breast tumors with multiple macroscopic nodules may have a different biology and a higher propensity to Lymph node metastasis, which is not simply a function of their tumor volume (16). This factor should be taken into account when evaluating the risk of Lymph node metastasis in breast cancer surgery.

A strong correlation between MIB-1 expression and tumor grade was also observed in this study. Tumor grading according to the modified Bloom-Richardson criteria is based on three major elements: the degree of glandular differentiation, relative nuclear pleomorphism, and mitotic activity (14). However, the assessment of these three factors entails some elements of 
subjectivity, thus involving incomplete inter-observer reproducibility, especially in intermediate-grade cases. The potential use of MIB-1 expression in the definition of a tumor grading system with a greater clinical utility has recently been suggested (21). We assessed the impact of MIB-1 expression on the risk of Lymph node metastasis in different grade subgroups amongst our patients and found a strong difference between MIB-1-negative and -positive cases in grade- 2 tumors. The risk of nodal metastasis in MIB-1-negative grade-2 cases was similar to grade-1 tumors, whereas the risk in MIB-1-positive grade- 2 cases was similar to grade- 3 tumors. This suggests that MIB-1 expression could identify subgroups of grade-2 tumors with a more aggressive clinical behaviour. During the St. Gallen Conference of 2001, the International Consensus Panel defined two risk categories for patients with nodenegative breast cancer: tumor grade 2 to 3 was one of the criteria for the inclusion in the high-risk category, in which adjuvant chemo-therapy is recommended (27). MIB-1 expression could be useful in distinguishing grade-2 tumors with a different biological aggressiveness, thus reducing problems related to doubtful pathological grade allocations.

In conclusion, the results of our prospective study indicate that the MIB-1 proliferation index may offer additional information about the risk of Lymph node metastasis in breast cancer, and could be useful in the identification of a more aggressive phenotype of grade-2 tumor. A strong relationship between Ki-67/MIB-1 expression and nodal status in pre-operative FNA biopsies has recently been reported (28). This may increase the clinical utility of this parameter in decision-making regarding the surgical approach for breast cancer patients.

\section{References}

1. Ravdin PM, de Laurentiis M, Vendely T and Clark GM: Prediction of axillary lymph node status in breast cancer patients by use of prognostic indicators. J Natl Cancer Inst 86: 1771-1775, 1994.

2. Brown DC and Gatter KC: Monoclonal antibody Ki-67: its use in histopathology. Histopathology 17: 489-503, 1990.

3. Gerdes J, Becker MH, Key G and Cattoretti G: Immunohistological detection of tumor growth fraction (Ki-67 antigen) in formalin-fixed and routinely processed tissues. J Pathol 168: 85-86, 1992.

4. Remmele W, Muhlfait V and Keul HG: Estimation of the proliferative activity of human breast cancer tissue by means of the Ki-67 and MIB-1 antibodies - comparative studies on frozen and paraffin sections. Virchows Arch 426: 435-439, 1995.

5. Orucevic A, Reddy VB, Bloom KJ, Bitterman P, Magi-Galluzzi C, Oleske DM, Phillips M, Gould VE, Cobleigh M, Wick MR and Gattuso P: Predictors of lymph node metastasis in T1 breast carcinoma, stratified by patient age. Breast J 8: 349-355, 2002.

6. Bader AA, Tio J, Petru E, Buhner M, Pfahlberg A, Volkholz H and Tulusan AH: T1 breast cancer: identification of patients at low risk of axillary lymph node metastases. Breast Cancer Res Treat 76: 11-17, 2002.

7. Isola JJ, Helin HJ, Helle MJ and Kallioniemi OP: Evaluation of cell proliferation in breast carcinoma. Comparison of Ki-67 immunohistochemical study, DNA flow cytometric analysis, and mitotic count. Cancer 65: 1180-1184, 1990.

8. Keshgegian AA and Cnaan A: Proliferation markers in breast carcinoma. Mitotic figure count, S-phase fraction, proliferating cell nuclear antigen, Ki-67 and MIB-1. Am J Clin Pathol 104: 42-49, 1995.

9. Seshadri R, Leong AS, McCaul K, Firgaira FA, Setlur V and Horsfall DJ: Relationship between p53 gene abnormalities and other tumor characteristics in breast-cancer prognosis. Int $\mathrm{J}$ Cancer 69: 135-141, 1996.
10. Spyratos F, Ferrero-Pous M, Trassard M, Hacene K, Phillips E, Tubiana-Hulin M and Le Doussal V: Correlation between MIB-1 and other proliferation markers: clinical implications of the MIB-1 cutoff value. Cancer 94: 2151-2159, 2002.

11. Pinto AE, Andre S, Pereira T, Nobrega S and Soares J: Prognostic comparative study of S-phase fraction and Ki-67 index in breast carcinoma. J Clin Pathol 54: 543-549, 2001.

12. Mirza AN, Mirza NQ, Vlastos G and Singletary SE: Prognostic factors in node-negative breast cancer: a review of studies with sample size more than 200 and follow-up more than 5 years. Ann Surg 235: 10-26, 2002.

13. Offersen BV, Sorensen FB, Knoop A, Overgaard J and the Danish Breast Cancer Cooperative Tumor Biology Committee: The prognostic relevance of estimates of proliferative activity in early breast cancer. Histopathology 43: 573-582, 2003.

14. Elston CW and Ellis IO: Pathological prognostic factors in breast cancer. I. The value of histological grade in breast cancer: experience from a large study with long-term follow-up. Histopathology 19: 403-410, 1991

15. Sobin LH and Wittekind C: TNM classification of malignant tumors. 6th edition. John Wiley \& Sons, New York, 2002.

16. Andea AA, Bouwman D, Wallis T and Visscher DW: Correlation of tumor volume and surface area with lymph node status in patients with multifocal/multicentric breast carcinoma. Cancer 100: 20-27, 2004.

17. Marrelli D, De Stefano A, de Manzoni G, Morgagni P, di Leo A and Roviello F: Prediction of recurrence after radical surgery for gastric cancer: a scoring system obtained from a prospective multicenter study. Ann Surg 241: 247-255, 2005.

18. Silverstein MJ, Gierson ED, Waisman JR, Colburn WJ and Gamagami P: Predicting axillary node positivity in patients with invasive carcinoma of the breast by using a combination of $\mathrm{T}$ category and palpability. J Am Coll Surg 180: 700-704, 1995.

19. Guarnieri A, Neri A, Correale PP, Lottini M, Testa M, Mariani F, Tucci E, Megha T, Cintorino M and Carli A: Prediction of lymph node status by analysis of prognostic factors and possible indications for elective axillary dissection in T1 breast cancers. Eur J Surg 167: 255-259, 2001

20. Mengel M, von Wasielewski R, Wiese B, Rudiger T, MullerHermelink HK and Kreipe H: Inter-laboratory and inter-observer reproducibility of immunohistochemical assessment of the Ki-67 labelling index in a large multi-centre trial. J Pathol 198: 292-299, 2002.

21. Trihia H, Murray S, Price K, Gelber RD, Golouh R, Goldhirsch A, Coates AS, Collins J, Castiglione-Gertsch M and Gusterson BA; International Breast Cancer Study Group: Ki-67 expression in breast carcinoma: its association with grading systems, clinical parameters, and other prognostic factors - a surrogate marker? Cancer 97: 1321-1331, 2003.

22. Gajdos C, Tartter PI and Bleiweiss IJ: Lymphatic invasion, tumor size, and age are independent predictors of axillary lymph node metastases in women with T1 breast cancers. Ann Surg 230: 692-696, 1999

23. Schoppmann SF, Bayer G, Aumayr K, et al: Austrian Breast and Colorectal Cancer Study Group. Prognostic value of lymphangiogenesis and lymphovascular invasion in invasive breast cancer. Ann Surg 240: 306-312, 2004.

24. Viale G, Maiorano E, Pruneri G, Mastropasqua MG, Valentini S, Galimberti V, Zurrida S, Maisonneuve P, Paganelli G and Mazzarol G: Predicting the risk for additional axillary metastases in patients with breast carcinoma and positive sentinel lymph node biopsy. Ann Surg 241: 319-325, 2005.

25. Buxant F, Anaf V, Simon P, Fayt I and Noel JC: Ki-67 immunostaining activity is higher in positive axillary lymph nodes than in the primary breast tumor. Breast Cancer Res Treat 75: 1-3, 2002.

26. Fish EB, Chapman JA and Link MA: Assessment of tumor size for multifocal primary breast cancer. Ann Surg Oncol 5: 442-446, 1998.

27. Goldhirsch A, Glick JH, Gelber RD, Coates AS and Senn HJ: Meeting highlights: International Consensus Panel on the Treatment of Primary Breast Cancer. Seventh International Conference on Adjuvant Therapy of Primary Breast Cancer. J Clin Oncol 19: 3817-3827, 2001.

28. Billgren AM, Tani E, Liedberg A, Skoog L and Rutqvist LE: Prognostic significance of tumor cell proliferation analyzed in fine needle aspirates from primary breast cancer. Breast Cancer Res Treat 71: 161-170, 2002. 